\title{
DESCRIPCIÓN DEL MECANISMO BIOQUÍMICO DE LA BIOHIDROGENACIÓN EN EL RUMEN DE ÁCIDOS GRASOS POLIINSATURADOS: UNA REVISIÓN
}

\section{DESCRIPTION OF THE BIOCHEMISTRY MECHANISM OF POLYUNSATURATED FATTY ACID RUMINAL BIOHYDROGENATION: A REVIEW}

\author{
Julián Castillo V. ${ }^{1}$, Martha Olivera A. ${ }^{2}$, Juan Carulla F. ${ }^{3}$ \\ ${ }^{1}$ Químico, M.Sc. Facultad de Medicina Veterinaria y de Zootecnia, Universidad Nacional de Colombia, Carrera 45 No. 26-85, \\ Bogotá D.C. Colombia. jcastillov@unal.edu.co. Autor para correspondencia: jcastillov@unal.edu.co; ${ }^{2}$ Médico Veterinario, \\ Dr. Sci. Agric. Facultad de Ciencias Agrarias, Universidad de Antioquía, Carrera 75 No. 65-87, Ciudadela Robledo, Medellín \\ Colombia, syngamia@gmail.com; ${ }^{3}$ Zootecnista, M.Sc, Ph.D. Facultad de Medicina Veterinaria y de Zootecnia, Universidad \\ Nacional de Colombia, jecarullaf@unal.edu.co
}

Rev. U.D.CA Act. \& Div. Cient. 16(2): 459-468, Julio-Diciembre, 2013

\section{RESUMEN}

La hidrólisis de los galactolípidos, los fosfolípidos, los sulfolípidos y los triglicéridos es el primer paso en el metabolismo de lípidos en el rumen, liberando ácidos grasos saturados, monoinsaturados y poliinsaturados. Recientemente, se ha reportado que el mecanismo de producción de ácido linoléico conjugado $\left(\mathrm{C}_{18: 2} \mathrm{cis}-9\right.$, trans- $\left.11, \mathrm{ALC}\right)$, está asociado a un desplazamiento prototrópico y que en la síntesis de su isómero $\mathrm{C}_{18: 2}$ trans-10, cis-12, participa la forma oxidada de flavin adenin dinucleótido (FAD). Primero, el ácido linoléico $\left(\mathrm{C}_{18: 2}\right.$ cis-9, cis-12, ALi) es convertido en ALC, que se transforma en ácido trans-vaccénico ( $\mathrm{C}_{18: 1}$ trans-11, ATV), produciéndose, finalmente, ácido esteárico. El ALi es el precursor del ALC y ATV, y el FAD es usado como cofactor en estos procesos. Una de las conclusiones de esta revisión es que a medida que el conocimiento del proceso de biohidrogenación aumenta, se dispone de más oportunidades para su aplicación. Una de las más relevantes es el diseño de estrategias nutricionales para incrementar la concentración de ácidos grasos bioactivos en leche y en carne.

Palabras clave: Ácido esteárico, ácido linoléico, ALC, ATV, enzimas.

\section{SUMMARY}

Hydrolysis of galactolipids, phospholipids, sulfolipids and triglycerides is the first step in ruminal lipid metabolism resulting in saturated, monounsaturated and polyunsaturated fatty acids. It has been reported recently that the mechanism of conjugated linoleic acid ( $\mathrm{C}_{18: 2}$ cis- 9 , trans- 11 , CLA) production is associated with a prototropic displacement and the synthesis of its isomer $\mathrm{C}_{18: 2}$ trans-10, cis-12, involves oxidated flavin adenine dinucleotide (FAD). First, linoleic acid $\left(\mathrm{C}_{18: 2}\right.$ cis-9, cis-12, LiA) is converted to CLA by the action of cis-9, trans-11 octadecanoate reductase, then CLA converts to trans-vaccenic acid $\left(\mathrm{C}_{18: 1}\right.$ trans-11, TVA) with the final product being stearic acid. LiA is the precursor of both CLA and TVA and FAD is used as a cofactor in these processes. One of the conclusions of this review is that as the knowledge of the ruminal biohydrogenation process increases, opportunities for its application become available. The nutritional strategies to increase the concentration of bioactive fatty acids in both milk and meat are the more relevant.

Key words: CLA, enzymes, linoleic acid, stearic acid, TVA.

\section{INTRODUCCIÓN}

Los ácidos grasos poliinsaturados (AGPI), particularmente, el ácido linoléico $\left(\mathrm{C}_{18: 2}\right.$ cis-9, cis-12, $\left.\mathrm{ALi}\right)$ y el ácido alfa-linolénico $\left(\mathrm{C}_{18: 3}\right.$ cis-9, cis-12, cis-15, ALn), se encuentran en altas proporciones en los lípidos de los forrajes y de algunos suplementos (Bauman et al. 1999; Kelly et al. 1998; Agazzi et al. 2004; Choi et al. 2009; Shen et al. 2011; Zened 
et al. 2013). Estos ácidos forman parte de la dieta de los rumiantes y dependiendo de su concentración en la dieta, modifican el perfil de ácidos grasos de la leche y la carne. La composición de ácidos grasos en la leche y en la carne de los rumiantes, se caracteriza por la presencia de una mayor concentración de ácidos grasos saturados que insaturados, debido al proceso de biohidrogenación (BH) en el rumen (Ashes et al. 1992; Bauman et al. 1999; Chow et al. 2004; Griinari \& Bauman, 1999; Dhiman et al. 2000; Abughazaleh \& Jacobson, 2007; Shen et al. 2011; Zened et al. 2013). Es por esto, que el conocimiento del proceso de $\mathrm{BH}$, se considera un punto crítico para modificar la relación entre ácidos grasos saturados e insaturados, en la leche y en la carne.

Se han estudiado diversos factores que afectan el proceso de $\mathrm{BH}$ del ALi y ALn, como también estrategias nutricionales que muestran resultados positivos en el incremento de ácido trans-vaccénico $\left(\mathrm{C}_{18: 1}\right.$ trans-11, ATV) y ácido linoléico conjugado $\left(\mathrm{C}_{18: 2}\right.$ cis-9, trans-11, ALC), en la leche y en la carne. Se ha reportado que estos compuestos tienen efectos potencialmente benéficos para la salud humana (Harfoot \& Hazlewood, 1997; O'shea et al. 1998; Khanal, 2004; Herrera et al. 2004; Perfield et al. 2007). En un trabajo in vitro, recientemente realizado en nuestro laboratorio, se encontró que la proporción, en la cual se combinan el ALi y ALn, puede afectar el proceso de $\mathrm{BH}$, modificando la proporción de los intermediarios (Castillo, J.; Pabón, M.; Olivera, M.; Daza, E.; Ribeiro, C.; Carulla, J, en proceso de elaboración para su publicación). En esta revisión, se busca presentar el conocimiento existente sobre el mecanismo bioquímico de la $\mathrm{BH}$ en el rumen, lo que permitiría diseñar estrategias nutricionales para incrementar la concentración de ácidos grasos poliinsaturados en la leche y en la carne. Esta revisión describirá el metabolismo de lípidos en el rumen y las principales rutas del proceso de biohidrogenación de los ácidos grasos insaturados.

\section{BIOQUÍMICA DEL PROCESO DE LIPÓLISIS}

Los lípidos de los forrajes contienen galactolípidos (mono y digalactosildiacilglicéridos) y, en una menor proporción, sulfolípidos (sulfoquinovosildiacilglicérido) y fosfolípidos (principalmente, fosfatidilcolina, fosfatidilglicerol y fosfatidiletanolamina) (Lee et al. 2007; Kaniuga, 2008; HalmemiesBeauchet-Filleau et al. 2013). Los cereales o los alimentos balanceados que contienen aceites vegetales son ricos en triacilgliceroles (Harfoot \& Hazlewood, 1997; Buccioni et al. 2006; AbuGhazaleh \& Jacobson, 2007; Albertí et al. 2013).

En rumiantes, el proceso de lipólisis lo realiza, principalmente, la especie bacteriana Anaerovibrio lipolítica, por la acción de sus lipasas asociadas a sus estructuras membranosas externas, presentándose así un mecanismo lipolítico, estrictamente extracelular (Hobson \& Summers, 1966; Hobson \& Summer, 1967; Enjalbert et al. 2003). Las lipasas tienen una actividad máxima enzimática a $\mathrm{pH}=7,4$ entre $20-22^{\circ} \mathrm{C}$, que se potencializa con el incremento de la concentración de $\mathrm{CaCl}_{2}$ y $\mathrm{BaCl}_{2}$ y que se inhibe con la presencia de $\mathrm{ZnCl}_{2}$ y $\mathrm{HgCl}_{2}$ (Henderson, 1971).

Aunque el proceso lipolítico no se atribuye enteramente a la especie mencionada, ya que existen reportes de otros microorganismos con actividad lipolítica, como lo son las especies bacteriales Butirivibrio fibrisolvens y Butirivibrio LM8/1B (Hassim et al. 2010), esta especie se considera como la más relevante en la lipólisis; el papel de los protozoos es reducido y con resultados muy variables y los hongos no intervienen en este proceso (Harfoot \& Hazlewood, 1997; Jenkins et al. 2008).

En la lipolisis, los galactoacilgliceroles se hidrolizan a glicerol, ácidos grasos y galactosa; los fosfolípidos, a ácidos grasos libres, fosfato y glicerol y los triacilgliceroles, a ácidos grasos y glicerol (Dawson et al. 1974; Jenkins et al. 2008; Kaniuga, 2008; Cabiddu et al. 2010; Halmemies-BeauchetFilleau et al. 2013).

El glicerol, se incorpora rápidamente a la glicólisis para la producción de piruvato, el cual, es posteriormente transformado en propionato, mediante el proceso de fermentación anaerobia (Jouany et al. 2007; Jenkins et al. 2008). La galactosa es rápidamente fermentada y transformada en ácidos grasos volátiles y los ácidos grasos mono y poliinsaturados, se biohidrogenan (Dawson et al. 1974; Hazlewood et al. 1976; Doreau et al. 2007).

Los monogalactosildiacilgliceroles, se hidrolizan más rápidamente que los digalactosildiacilgliceroles (Dawson \& Hemington, 1974; Dawson et al. 1974; Cabiddu et al. 2010) y los diacilgliceroles, se hidrolizan más rápidamente que los triacilgliceroles (Harfoot \& Hazlewood, 1997; Shen et al. 2011). La velocidad de lipólisis aumenta cuando se incrementa el grado de insaturación de los ácidos grasos, con la excepción de los ácidos grasos presentes en los aceites de pescado (Harfoot \& Hazlewood, 1997; Jenkins, 1993; AbuGhazaleh \& Jenkins, 2004).

\section{BIOQUÍMICA DEL PROCESO DE BIOHIDROGENACIÓN}

Hace aproximadamente 81 años, se encontró que los lípidos que forman los tejidos de los rumiantes eran más saturados que los de los no rumiantes (Banks \& Hilditch, 1931) y, por muchos años, se creyó que el proceso de $\mathrm{BH}$ de los lípidos ocurría en los tejidos. En la actualidad, se sabe que este pro- 
ceso ocurre en el rumen por acción de los microorganismos y, en una pequeña proporción, en el tracto intestinal posterior (Harfoot \& Hazlewood, 1997; Lee \& Jenkins, 2011).

Wright (1959; 1960) encontró que las bacterias son los microorganismos más importantes en el proceso de BH, lo cual, también es concluido por Dawson \& Kemp (1969) y Singh \& Hawke (1979). En otros estudios, se halló que, aproximadamente, la mitad de los microorganismos involucrados en la digestión de los lípidos se encuentran asociados a la porción líquida del rumen (BAL) y los restantes se hallaban adheridos a las superficies del alimento (BAS) (Hungate, 1966).

Utilizando contenidos ruminales, se evidenció que la presencia de partículas del alimento aumenta la velocidad del proceso de biohidrogenación (Herrera et al. 2004). Martin \& Valeille (2002) notaron que el proceso de BH del ALi en el rumen ocurre por la adhesión del ALi a las partículas de alimento.

Legay-Carmier \& Bauchart (1989) encontraron que en una dieta para vacas suplementada con aceite de soya, el $70 \%$ en masa de las bacterias eran BAS y, solamente un 7\%, BAL; el restante $23 \%$ era de bacterias pobremente adheridas a la superficie o se transferían, de manera constante, desde las partículas al medio líquido ruminal. Bauchart et al. (1990) mostraron que existe "preferencia" por parte de los ácidos grasos a adherirse a las partículas de alimento y que la concentración total de los ácidos grasos que se adherían a las BAS era casi del doble, con respecto al del BAL.

Se puede concluir que después de la lipólisis y de la liberación de ácidos grasos, éstos se adhieren a las partículas sólidas de alimento y son hidrogenados de manera preferencial por las BAS, pudiéndose generar competencia entre las partículas y las bacterias.

Descripción del mecanismo de la biohidrogenación de los ácidos grasos insaturados: La lipólisis constituye un paso obligado antes de la $\mathrm{BH}$ y las bacterias son las principales responsables del proceso de $\mathrm{BH}$, aunque los hongos y los protozoos pueden participar en la biohidrogenación (Harfoot \& Hazlewood, 1997; Yañez-Ruiz et al. 2006; Maia et al. 2007; Váradyová et al. 2008; Buccioni et al. 2012).

Sachan \& Davis (1969) hallaron que la especie Borrelia B25 biohidrogenaba el $\mathrm{ALi}$, pero no el ácido oléico $\left(\mathrm{C}_{18: 1} \mathrm{cis}-9\right.$, AOl). Kemp et al. (1975) registraron tres especies bacteriales implicadas en la biohidrogenación de los ácidos grasos insaturados, siendo estas Ruminococcus albus, Eubacterium y Fusocillus.

La especie Fusocillus biohidrogenó el AOl y ALi hasta ácido esteárico $\left(\mathrm{C}_{18: 0}\right)$ y el ALn hasta $\mathrm{C}_{18: 1}$ cis-15. La especie $R$. al- bus y la de Eubacterium no biohidrogenaron el AOl, pero sí convirtieron el ALi y ALn en una mezcla de ácidos octadecenóicos, donde el ATV fue el isómero predominante.

Harfoot \& Hazlewood (1997) y Maia et al. (2010) ilustran el papel de una gran variedad de microorganismos que participan en el proceso de BH. Otros trabajos, como los de Maia et al. (2007; 2010) y Jenkins et al. (2008) presentan una caracterización reciente del ambiente microbiológico ruminal y reportan la toxicidad de diferentes niveles de inclusión de ácidos grasos mono y poliinsaturados, sobre el crecimiento de las especies implicadas en el proceso de biohidrogenación.

La identificación de los intermediarios producidos y de los diferentes microorganismos ruminales que participan en el proceso de $\mathrm{BH}$ ruminal, ha permitido establecer su mecanismo (Bauman et al. 1999; Lee \& Jenkins, 2011; Buccioni et al. 2012). El proceso de BH involucra varios pasos bioquímicos, con velocidades, intermediarios y especies bacteriales características (Bauman et al. 1999). Lourenço et al. (2010) presentan una descripción general del amplio número ácidos grasos, resultantes de la BH del ALi y ALn.

Para los pasos principales, Kemp \& Lander (1984) dividieron las bacterias en dos grupos, considerando las reacciones químicas en que intervenían y los productos de la $\mathrm{BH}$. El grupo A es el responsable de transformar el ALi hasta el ATV; por su parte, el grupo B transforma el ATV en $\mathrm{C}_{18: 0}$. Para el ALn, la $\mathrm{BH}$ es más compleja e involucra los dos grupos de bacterias en todos los pasos (Figura 1). Ambos mecanismos presentan, como paso inicial, la isomerización del enlace cis-12, de lo cual, resulta la formación de un intermediario químico con un sistema conjugado con isomería geométrica cis-9, trans-11 (Harfoot \& Hazlewood, 1997; Buccioni et al. 2012). Esto está de acuerdo con lo evidenciado en un estudio realizado en nuestro laboratorio, en donde se evaluó el efecto de la combinación de diferentes proporciones de ALi y ALn, sobre su $\mathrm{BH}$ y la concentración de los principales intermediarios generados, a partir de dichos ácidos grasos (Castillo, J.; Pabón, M.; Olivera, M.; Daza, E.; Ribeiro, C.; Carulla, J., en proceso de elaboración para su publicación), apreciando que se produce un incremento en la concentración de ALC, en función del aumento de la relación ALi:ALn.

En estudios con Butirivibrio fibrisolvens, se ha establecido que el primer paso es realizado por la enzima linoleato isomerasa (EC 5.2.1.5), que se encuentra en la membrana de las bacterias (Jenkins et al. 2008). Kepler et al. (1971) propusieron, por primera vez, un mecanismo de reacción, definiendo que existen dos sitios de interacción entre la molécula de ALi y la enzima, los cuales, son el sistema de electrones $\pi$ (cis-9, cis-12) y el grupo carboxilo. Al presentarse la interacción de estas regiones con la enzima, se produce una conformación definida de la molécula de ALi y así, se puede transferir un 


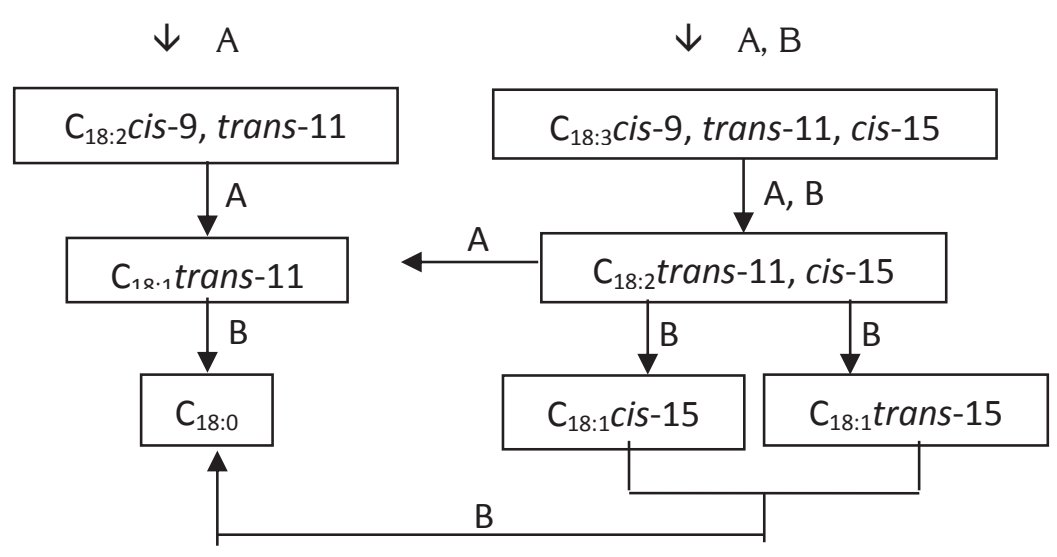

Figura 1. Rutas principales de la biohidrogenación del ácido linoléico y alfa-linolénico en el rumen, junto con los grupos de microorganismos implicados (Harfoot E Hazlewood, 1997; Bucccioni et al. 2012). Las letras A y B indican los dos grupos bacteriales implicados en el proceso.

protón de una tercera región donante de la enzima al carbono 13 , haciendo que éste adquiera una configuración $\mathrm{D}$, con lo cual, se produce un compuesto con el sistema conjugado cis-9, trans-11. Kepler et al. (1971) demostraron que para que se lleve a cabo el proceso de $\mathrm{BH}$, el grupo carboxilo del ácido graso debe estar libre.

Cuando la linoleato isomerasa se incubó con ALi en presencia de ${ }^{2} \mathrm{H}_{2} \mathrm{O}$, un átomo de ${ }^{2} \mathrm{H}$ se incorporó resultando en la formación del ALC en una configuración R (grado de marcación, 88\%); esto sugiere, que el mecanismo de isomerización es de desplazamiento prototrópico. Mediante el uso de ALi deuterado en la posición 11, como sustrato para esta enzima, se mostró que el mecanismo de isomerización de la linoleato isomerasa de Butirivibrio fibrisolvens involucra la remoción del hidrógeno ubicado en el carbono 11 y su transferencia en la misma molécula, ya que no se detectó su pérdida (Liavonchanka \& Feussner, 2008).

Los mecanismos de isomerización mejor conocidos son los de las isomerasas producidas por Butirivibrio fibrisolvens (IBF), Propionobacterium filicina (IPF) y P. acnes (IPA) (Liavonchanka \& Feussner, 2008). Se tiene conocimiento para el ALi, que la IPA transfiere un hidrógeno del carbono 11 con estereoquímica $\mathrm{R}$ al carbono 9 , con la misma estereoquímica R.

La IPF transfiere un hidrógeno del carbono 11 con estereoquímica $S$ a la posición 13 , cuya estereoquímica se desconoce y, para la IBF, ocurre el proceso inverso, es decir, la estereoquímica del protón retirado de la posición 11 es desconocida, pero la del agregado en el carbono 13 presenta estereoquímica R (Liavonchanka et al. 2006).
Liavonchanka et al. (2006) estudiaron la estructura y el mecanismo de la isomerasa de AGPI de $P$. acnes, estableciendo que el proceso catalítico de conversión del ALi en $C_{18: 2}$ trans-10, cis-12 (que se produce cuando se administran dietas altas en almidón) (Halmemies-Beauchet-Filleau et al. 2013) (Figura 2), se realiza por medio del cofactor Flavin Adenin Dinucleótido (FAD), mediante la abstracción de un hidruro del carbono 11 del ALi en configuración R, produciéndose un carbocatión estabilizado por resonancia, en el cual, se realiza la isomerización del doble enlace cis de la posición 9, a la posición 10 con geometría trans, que produce un segundo carbocatión, cuya carga se ubica en el carbono 9, posición donde es transferido el hidruro inicialmente removido, produciéndose el isómero $\mathrm{C}_{18: 2}$ trans-10, cis-12 (Liavonchanka \& Feussner, 2008).

Termodinámicamente, la isomerización de $\mathrm{ALi}$ en $\mathrm{C}_{18: 2}$ trans-10, cis-12 es un proceso que demanda energía, porque se requiere la ruptura de un enlace $\mathrm{C}-\mathrm{H}$, como paso previo a la isomerización del doble enlace. Para la formación del intermediario alílico, se requiere, aproximadamente, $+16,7 \mathrm{~kJ} . \mathrm{M}^{-1}$, haciendo de esta reacción, un proceso termodinámicamente irreversible (Liavonchanka \& Feussner, 2008). Aunque no se tiene claro cómo es suministrada esta energía para las IBF e IPF, para la IPA se sabe que la activación y la transferencia del hidrógeno de la posición 11, es mediada por el FAD. La energía libre de Gibbs $(\Delta \mathrm{Grxn})$ a $37^{\circ} \mathrm{C}$, para la isomerización del ALi $(-4,856 \mathrm{kcal} / \mathrm{mol})$ y ALn $(-754 \mathrm{kcal} /$ mol), a sus intermediarios conjugados, permite definir que dichos procesos se constituyen como termodinámicamente favorables, según los valores calculados en nuestro laboratorio (Castillo, J.; Pabón, M.; Olivera, M.; Daza, E.; Ribeiro, C.; Carulla, J., en proceso de elaboración para su publicación). 


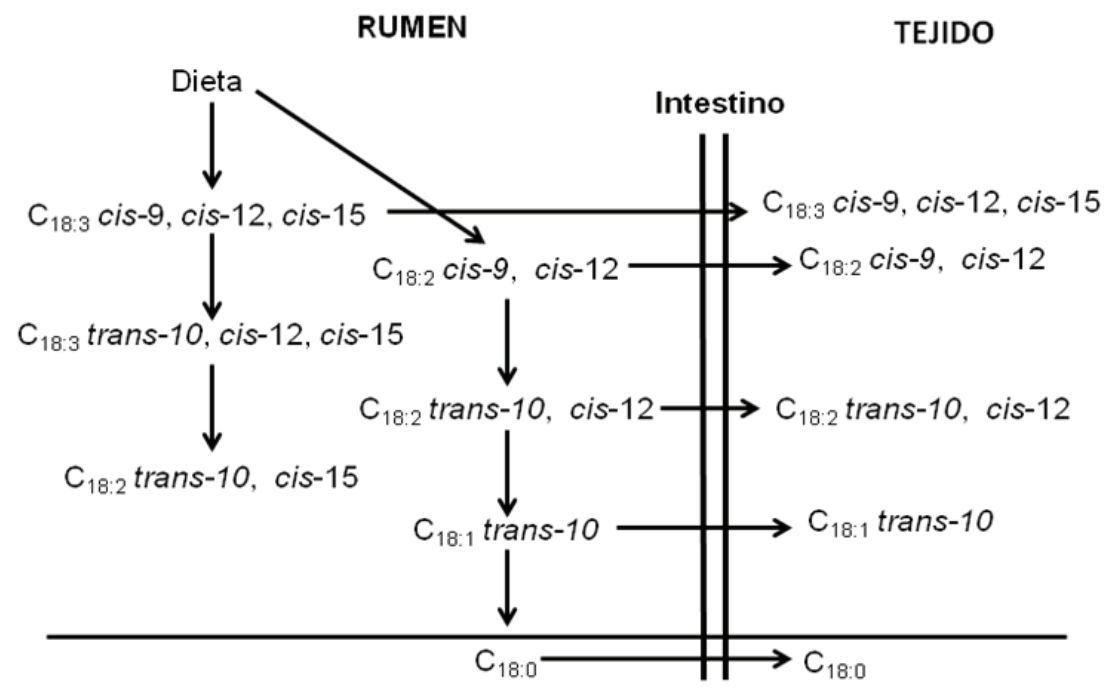

Figura 2. Biohidrogenación de ácidos grasos insaturados en el rumen, a pH bajo (adaptado de Buccioni et al. 2012).

La IBF a diferencia de la IPA realiza un intercambio de un protón entre el solvente y el isómero $\mathrm{C}_{18: 2}$ cis-9, trans-11. Liavonchanka \& Feussner (2008) propusieron que la geometría del sitio activo de la trans-10, cis-12 isomerasa podría ser diferente al de la cis-9, trans-11 isomerasa, argumentando la posible presencia de agua o residuos de aminoácidos polares en el sitio activo de las isomerasas.

Así, la protonación del ALi en la posición 13 podría ser mediada por el nitrógeno 5 del FAD, considerando que al formar un grupo amino terciario, éste tiene la capacidad de intercambiar rápidamente protones con el solvente e incorporar deuterio $\left({ }^{2} \mathrm{H}\right)$ en el isómero $\mathrm{C}_{18: 2}$ cis-9, trans-11 (Wallace et al. 2007).

Kemp et al. (1984a) evaluaron la BH de isómeros geométricos de ácidos grasos de 18 carbonos, con dos dobles enlaces conjugados en diferentes posiciones, utilizando uno de los grupos de bacterias A o B. De todos los isómeros posibles, el único que no fue alterado estructuralmente fue el isómero cis-14, cis-17. Además, se encontró que los isómeros cis-2, cis-5 y cis-9, cis-12, fueron los únicos que se isomerizaron en sus correspondientes estructuras cis, trans, antes de ser biohidrogenados. McKain et al. (2010) evaluaron el proceso de $\mathrm{BH}$ de diferentes isómeros posicionales y geométricos del ALC y AOl en óxido de deuterio $\left(\mathrm{D}_{2} \mathrm{O}\right)$ al $37,4 \%$, mediante el uso de Butirivibrio fibrisolvens JW11, $B$. proteoclastus P-18 y $P$. acnés.

Teniendo en cuenta que durante la incubación de los isómeros geométricos con insaturaciones en la posiciones 9 y 11, el enriquecimiento con deuterio de los intermediarios formados fue alrededor de la mitad del de los isómeros $\mathrm{C}_{18: 2}$ trans-10, cis-12 y AOl, los autores sugieren que la reducción de los isómeros geométricos 9, 11 ocurre mediante un mecanismo diferente al de los otros ácidos grasos insaturados (Wallace et al. 2007).

El mecanismo de formación de los isómeros del ALC a partir de ALi, utilizando contenido ruminal de ovejas o cultivos de microorganismos puros de $B$. fibrisolvens y $C$. proteoclasticum, mostraron que los isómeros del ALC, $\mathrm{C}_{18: 2}$ trans-9, trans-11 y $\mathrm{C}_{18: 2}$ trans-10, cis-12 eran los que se producían en mayor cantidad y se encontraron trazas de $\mathrm{C}_{18: 2}$ trans-9, cis-11, $\mathrm{C}_{18: 2}$ cis-9, cis-11 y C 18:2 $_{2}$ cis-10, cis-12 (Wallace et al. 2007).

Wallace et al. (2007) sugirieron que el mecanismo de biosíntesis del ALC, se inicia con la abstracción del $\mathrm{H}$ del carbono 11 del ALi, formándose un radical termodinámicamente inestable, en el cual, se produce la translocación del doble enlace, de la posición 12 a la posición 11, con cambio de geometría cis a trans, con lo que se forma un radical, cuyo electrón desapareado se ubica en el carbono 13. A partir de marcación isotópica y mediante espectrometría de masas, se sugirió que los isómeros geométricos del ALC que presentaban insaturaciones en las posiciones 10 y 12 , eran sintetizados por un mecanismo que difiere de la síntesis de los isómeros 9, 11.

Finalmente, un átomo de hidrógeno es proporcionado por una molécula de agua al carbono 13, para producir ALC.

A partir del mecanismo propuesto, Wallace et al. (2007) 
afirmaron que las diferencias en concentración encontradas para los isómeros del ALC, se podrían sustentar por la formación de intermediarios energéticamente menos favorables. Lo anterior es claramente coincidente con los resultados encontrados por Kemp et al. (1984a) y podría dar una explicación parcial a los resultados, donde se evalúo la cinética de BH de ALi y ALn y la generación de intermediarios y productos (Chouinard et al. 2001; Troegeler-Meynadier et al. 2003; Ribeiro et al. 2007; Shingfield et al. 2010; Prieto et al. 2013).

El segundo paso en el proceso de $\mathrm{BH}$ es la reducción del enlace cis-9 del sistema conjugado, para producir el ATV a partir del ALi y el $\mathrm{C}_{18: 2}$ trans-11, cis-15 a partir del ALn. Este paso involucra la adición de dos hidrógenos al enlace cis-9 del sistema dieno conjugado cis-9, trans-11, por la enzima cis-9, trans-11 octadecadienoato reductasa (EC 1.3.1.-), la cual, ha sido aislada y purificada de $B$. fibrisolvens (Hughes et al. 1982; Jenkins et al. 2008; Sterk et al. 2010).

La enzima es una glicoproteína con 10 moles de fucosa y 12 de galactosa por mol de enzima y que presenta $\mathrm{Fe}^{3+}$ coordinado, indispensable para su actividad enzimática y se encuentra involucrado directamente en el proceso de reducción (Harfoot \& Hazlewood, 1997; Buccioni et al. 2012).

El segundo paso de la $\mathrm{BH}$ en el rumen, ha sido estudiado para la especie $B$. fibrisolvens (Rosenfeld \& Tove, 1971; Lee \& Jenkins, 2011). Yamazaki \& Tove (1979) aislaron, a partir de $B$. fibrisolvens, un electrodonor para la $\mathrm{BH}$ del enlace cis-9, que fue identificado como alfa-tocoferolquinol $\left(\mathrm{TQH}_{2}\right)$ (Hughes \& Tove, 1980a). Los mismos autores (1980b), sugirieron que dos moléculas de $\mathrm{TQH}_{2}$ fueron oxidadas a dos semiquinonas (TQH), aportando así cada una, un electrón para la reducción del enlace cis del sistema conjugado.

Estudios in vitro usando ALi marcado mostraron que la isomerización del enlace cis-12 involucra la rápida $\mathrm{BH}$ del ALC hasta ATV. La BH del ATV (tercer paso de la BH) ocurre más lentamente y, por lo tanto, se acumula y puede aumentar su disponibilidad para su absorción (Singh \& Hawke, 1979; Moate et al. 2008; Buccioni et al. 2012). Experimentos in vitro en nuestro laboratorio, sugirieron que la proporción de ALi y ALn en una mezcla alteró la concentración de ALi y de diversos intermediarios y productos (Castillo J., Pabón M., Olivera M., Daza E., Ribeiro C., Carulla J, en proceso de elaboración para su publicación).

Adicionalmente, las tasas de conversión de ALi a ácido esteárico, para diferentes relaciones de ALi y ALn, eran menores que las de isomerización de ALi y ALn, donde una mayor proporción de ALi en la mezcla producía una mayor acumulación de ATV (Castillo, J.; Pabón, M.; Olivera, M.; Daza, E.; Ribeiro, C.; Carulla, J., en proceso de elaboración para su publicación).
Estos hallazgos son coherentes con lo hallado por Kemp et al. (1984b), quienes evaluaron la habilidad de la especie Fusocillus (perteneciente al grupo B), para hidrogenar diferentes isómeros de ácidos octadecenoicos, hasta $\mathrm{C}_{18: 0}$. Empleando diferentes isómeros cis, desde el cis- 2 hasta el cis-13 (excepto el cis-3) y todos los isómeros trans, desde el trans-2 hasta el trans-13 (excepto el trans-3 y trans-4), encontraron que se registraron conversiones a $\mathrm{C}_{18: 0}$, en un rango entre 73 y 79\%, para los isómeros cis, del cis-3 al cis-11 y, aproximadamente el 45\%, de los isómeros trans-8, trans-9 y trans-10 fueron hidrogenados.

La reducción del ATV parece ser el paso determinante de la $\mathrm{BH}$ de ALi y ALn y, por lo tanto, este intermediario se podría acumular en el rumen (Moate et al. 2008), aumentándose así su disponibilidad para ser absorbido.

Conclusiones: Esta revisión muestra que la BH del ácido linoléico y alfa-linolénico se realiza por etapas sucesivas de biohidrogenación e isomerización, con características químicas y termodinámicas distintivas y por acción de especies microbianas diferentes, donde el producto final es el ácido esteárico. Las rutas metabólicas de la $\mathrm{BH}$ del ácido alfa-linolénico son más extensas, lo que sugiere que este ácido produce un mayor número de compuestos que pueden aparecer en la biohidrogenación de los ácidos grasos, como el ALC y el ATV. Por el contrario, el ácido linoléico presenta un menor número de vías de biohidrogenación, lo que indicaría que ocurre por la ruta principal y las secundarias.

Conflicto de intereses: El manuscrito fue preparado y revisado con la participación de todos los autores, quienes declaramos que no existe ningún conflicto de intereses que ponga en riesgo la validez de los resultados presentados. Financiación: Este trabajo fue financiado por el Departamento Administrativo de Ciencia, Tecnología e Innovación de Colombia (Colciencias) y la División de Investigación de Bogotá (DIB) de la Universidad Nacional de Colombia.

\section{BIBLIOGRAFÍA}

1. ABUGHAZALEH, A.A.; JACOBSON, B.N. 2007. The effect of $\mathrm{pH}$ and polyunsaturated $\mathrm{C} 18$ fatty acid source on the production of vaccenic acid and conjugated linoleic acid in ruminal cultures incubated with docosahexaenoic acid. Anim. Feed Sci. Technol. 136:11-22.

2. ABUGHAZALEH, A.A.; JENKINS, T.C. 2004. Disappearance of Docosahexaenoic and Eicosapentaenoic acids from cultures of mixed ruminal microorganisms. J. Dairy Sci. 87:645-651. 
3. AGAZZI, A.; BAYOURTHE, C.; NICOT, M.C.; TROEGELERMEYNADIER, A.; MONCOULON, R.; ENJANBERT, P. 2004. In situ ruminal biohydrogenation of fatty acids from extruded soybeans: effects of dietary adaptation and of mixing with lecithin or wheat straw. Anim. Feed Sci. Technol. 117:165-175.

4. ALBERTÍ, P.; GÓMEZ, I.; MENDIZABAL, J.A.; RIPOLL, G.; BARAHONA, M.; SARRIÉS, V.; INSAUSTI, K.; BERIAIN, M.J.; PURROY, A.; REALINI, C. 2013. Effect of whole linseed and rumen-protected conjugated linoleic acid enriched diets on feedlot performance, carcass characteristics, and adipose tissue development in young Holstein bulls. Meat Sci. 94:208-214.

5. ASHES, J.R.; SIEBERT, B.D.; GULATI, S.K.; CUTHBERTSON, A.Z.; SCOTT, T.W. 1992. Incorporation of n-3 fatty acids of fish oil into tissue and serum lipids of ruminants. Lipids. 27:629-631.

6. BANKS, A.; HILDITCH, T.P. 1931. The Glyceride structure of beef talows. Biochem. J. 25:1168-1182.

7. BAUCHART, D.; LEGAY-CARMIER, F.; DOREAU, M., GAILLARD, B. 1990. Lipid metabolism of liquid-associated and solid-adherent bacteria in rumen contents of dairy cows offered lipid supplemented diet. Br. J. Nutr. 63:563-578.

8. BAUMAN, D.E.; BAUMGARD, L.H.; CORL, B.A.; GRIINARI, J.M. 1999. Biosynthesis of conjugated linoleic acid in ruminants. J. Anim Sci. 77:1-15.

9. BUCCIONI, A.; ANTONGIOVANNI, M.; PETACCHI, F.; MELE, M.; SERRA, A.; SECCHIARI, P.; BENVENUTI, D. 2006. Effect of dietary fat quality on C18:1 fatty acids and conjugated linoleic acid production: An in vitro rumen fermentation study. Anim. Feed Sci. Technol. 127:268-282.

10. BUCCIONI, A.; DECANDIA, M.; MINIERI, S.; MOLLE, G.; CABIDDU, A. 2012. Lipid metabolism in the rumen: New insights on lipolysis and biohydrogenation with an emphasis on the role of endogenous plant factors. Anim. Feed Sci. Technol. 174:1-25.

11. CABIDDU, A.; SALIS, L.; TWEED, J.K.S.; MOLLE, G.; DECANDIA, M.; LEE, M.R.F. 2010. The influence of plant polyphenols on lipolysis and biohydrogenation in dried forages at different phenological stages: in vitro study. J. Sci. Food Agric. 90:829-835.

12. CHOUINARD, P.Y.; CORNEAU, L.; BUTLER, B.R.; CHI-
LLIARD, Y.; DRACKLEY, J.K.; BAUMAN, D.E. 2001. Effect of dietary lipid source on conjugated linoleic acid concentration in milk fat. J. Dairy Sci. 84:680690.

13. CHOI, N.; PARK, H.G.; KIM, J.H.; HWANG, H.; KWON, K.H.; YOON, J.A.; KWON, E.G.; CHANG, J.; HWANG, I.H.; KIM, Y.J. 2009. Characterization of environmental factors in Conjugated Linoleic Acid Production by mixed rumen bacteria. J. Agric. Food Chem. 57:9263-9267.

14. CHOW, T.T.; FIEVEZ, V.; MOLONEY, A.P.; RAES, K.; DEMEYER, D.; SMET, S. 2004. Effect of fish oil in vitro rumen lipolysis, apparent biohydrogenation of linoleic and linolenic acid and accumulation of biohydrogenation intermediated. Anim. Feed Sci. Technol. 117:1-12.

15. DAWSON, R.M.C.; KEMP, P. 1969. The effect of defaunation on the phospholipids and on the hydrogenation of unsaturated fatty acids in the rumen. Biochem. J. 115:351-352.

16. DAWSON, R.M.C.; HEMINGTON, N. 1974. Digestion of grass lipids and pigments in the sheep rumen. $\mathrm{Br}$. J. Nutr. 32:327-340.

17. DAWSON, R.M.C.; HEMINGTON, N.; GRIME, D.; LANDER, D.; KEMP, P. 1974. Lipolysis and hydrogenation of galactolipids and the accumulation of phytanic acid in the rumen. Biochem. J. 144:169-171.

18. DHIMAN, T.R.; SATTER, L.D.; PARIZA, M.W.; GALLI, M.P.; ALBRIGHT, K.; TOLOSA, M.X. 2000. Conjugated linoleic acid (ALC) content of milk from cows offered diets rich in linoleic and linolenic acid. J. Dairy Sci. 83:1016-1027.

19. DOREAU, M.; REARTE, D.; PORTELLI, J.; PEYRAUD, J.L. 2007. Fatty acid ruminal metabolism and digestibility in cows fed perennial ryegrass. Eur. J. Lip. Sci. Technol. 109:790-798.

20. ENJALBERT, F.; EYNARD, P.; NICOT, M.C.; TROEGELERMEYNADIER, A.; BAYOURTHE, C.; MOCOULON, R. 2003. In vitro versus in situ ruminal biohydrogenation of unsaturated fatty acids from raw or extruded mixture of ground canola seed/canola meal. J Dairy Sci. 86:351-359.

21. GRIINARI, J.M.; BAUMAN, D.E. 1999. Biosynthesis of conjugated linoleic acid and its incorporation into meat and milk in ruminants. In: Yurawecs, M.P.; Mos- 
soba, M.M.; Kramer, J.K.; Pariza, M.W.; Nelson, G.J.; (eds.). Advanced in conjugated linoleic acids reseach Vol. 1 Champaign (IL): AOCS Press. p.180-200.

22. HALMEMIES-BEAUCHET-FILLEAU, A., KAIRENIUS, P.; AHVENJÄRVI, S.; CROSLEY, L.K.; MUETZEL, S.; HUHTANEN, P.; VANHATALO, A.; TOIVONEN, V.; WALLACE, R.J.; SHINGFIELD, K.J. 2013. Effect of forage conservation method on ruminal lipid metabolism and microbial ecology in lactating cows fed diets containing a 60:40 forage-to-concentrate ratio. J. Dairy Sci. 96:2428-2447.

23. HARFOOT, C.G.; HAZLEWOOD, G.P. 1997. Lipid metabolism in the rumen. In: Hobson, P.N.; Stewart, C.S. (eds).The Rumen Microbial Ecosystem, ed. Chapman and Hall, London, UK. p.382-426.

24. HASSIM, H.A.; LOURENÇO, M.; GOEL, G.; VLAEMINCK, B.; GOH, Y.M.; FIEVEZ, V. 2010. Effect of different inclusion levels of oil palm fronds on in vitro rumen fermentation pattern, fatty acid metabolism and apparent biohydrogenation of linoleic and linolenic acid. Anim Feed Sci. Technol. 162:155-158.

25. HAZLEWOOD, G.P.; KEMP, P.; LANDER, D.; DAWSON, R.M.C. 1976. C18 unsaturated fatty acid biohydrogenation patterns of some rumen bacteria and their ability to hydrolyse exogenous phospholipids. Br. J. Nutr. 35:293-297.

26. HENDERSON, C. 1971. A Study of the Lipase Produced by Anaerovibrio Lipolytica, a Rumen Bacterium. J. Gen. Microbiol. 65:81-89.

27. HERRERA, J.A.; SHAHABUDIN, A.K.M.; FAISAL, M.; ERSHENG, G.; WEI, J.; LIXIA, D.; GANDAHO, T.; LOPEZ, P. 2004. Efectos de la suplementación oral con Calcio y ácido linoléico conjugado en primigrávidas de alto riesgo. Colombia Médica. 35(1):1-8.

28. HOBSON, P.N.; SUMMERS, K. 1966. Effect of growth rate on the lipase activity of a rumen bacterium. Nature. 209:736-737.

29. HOBSON, P.N.; SUMMER, R. 1967. The continuos culture of anaerobic bacteria. J. Gen. Microbiol. 47:5365.

30. HUGHES, P.E.; TOVE, S.B. 1980a. Identification of an endogenous electron donor for biohydrogenation as $\alpha$-tocopherolquinol. J. Biol. Chem. 255:4447-4452.

31. HUGHES, P.E.; TOVE, S.B. 1980b. Identification of deoxy- $\alpha$-tocopherolquinol as another endogenous electron donor for biohydrogenation. J. Biol. Chem. 255:11802-11806.

32. HUGHES, P.E.; HUNTER, W.J.; TOVE, S.B. 1982. Biohydrogenation of unsaturated fatty acids. Purification and properties of cis-9, trans-11-octadecadienoate reductase. J. Biol. Chem. 257(1):3643-3649.

33. HUNGATE, R.E. 1966. The rumen and its microbes. New York academic press, New York, NY, USA. p.315-328.

34. JENKINS, T.C. 1993. Lipid metabolism in the rumen. J. Dairy Sci. 76:3851-3863.

35. JENKINS, T.C.; WALLACE, R.J.; MOATE, P.J.; MOSLEY, E.E. 2008. Recent advances in biohydrogenation of unsaturated fatty acids within the rumen microbial ecosystem. J. Anim. Sci. 86: 397-412.

36. JOUANY, J.P.; LASSALAS, B.; DOREAU, M.; GLASSER, F. 2007. Dynamic features of the rumen metabolism of linoleic acid, linolenic acid and linseed oil measured in vitro. Lipids. 42: 351-360.

37. KANIUGA, Z. 2008. Chilling response of plants: importance of galactolipase, free fatty acids and free radicals. Plant Biol. 10:171-184.

38. KELLY, M.L.; KOLVER, E.S.; BAUMAN, D.E.; VAN AMBURGH, M.E.; MULLER, L.D. 1998. Effect of intake of pasture on concentrations of conjugated linoleic acid in milk of lactating dairy cows. J. Dairy Sci. 81:1630-1636.

39. KEMP, P.; LANDER, D.J. 1984. The hydrogenation of the series of methylene-interrupted cis,cis-octadecadienoic acids by pure cultures of six rumen bacteria. $\mathrm{Br}$. J. Nutr. 52:171-177.

40. KEMP, P.; LANDER, D.J.; HOLMAN, R.T. 1984a. The hydrogenation of the series of methylene-interrupted cis, cis-octadecadienoic acid by pure cultures of rumen bacteria. Br. J. Nutr. 52:171-177.

41. KEMP, P.; LANDER, D.J.; GUNSTONE, F.D. 1984b. Hydrogenation of some cis and trans octadecenoic acid to stearic acid by a rumen Fusocillus sp. Br. J. Nutr. 52:165-170.

42. KEMP, P.; WHITE, R.W.; LANDER, D.J. 1975. The hydrogenation of insaturated fatty acids by five bacterial isolates from the sheep rumen, including a new species. J. Gen. Microbiol. 90:100-114. 
43. KEPLER, C.R.; TUCKER, W.P.; TOVE, S.B. 1971. Biohydrogenation of unsaturated fatty acids. V. Stereoespecificity of proton addition and mechanism of action of linoleic acid $\Delta 12$-cis, $\Delta 11$-trans- isomerase from butyrivibrio fibrisolvens. J. Biol. Chem. 246:2765-2771.

44. KHANAL, R.C. 2004. Potential health beneficts of conjugated linoleic acid (CLA): A review. AsianAustralasian J. Anim. Sci. 17(9):1315-1328.

45. LEE, M.R.F.; PAFITT, L.J.; SCOLLAN, N.D.; MINCHIN, F.R. 2007. Lipolysis in red clover with different polyphenol oxidase activities in the presence and absence of rumen fluid. J. Sci. Food Agric. 87:13081314.

46. LEE, Y.; JENKINS, T.C. 2011. Biohydrogenation of linolenic acid to stearic acid by the rumen microbial population yields multiple intermediate conjugated diene isomers. J. Nutr. 141(8):1445-1450.

47. LEGAY-CARMIER, F.; BAUCHART, D. 1989. Distribution of bacteria in the rumen contents of dairy cows given a diet supplemented with soya-bean oil. Br. J. Nutr. 61:725-740.

48. LIAVONCHANKA, A.; HORNUNG, E.; FEUSSNER, I.; RUDOLPH, M.G. 2006. Structure and mechanism of the Propionibacterium acnes polyunsaturated fatty acid isomerase. Proc. Nal. Acad. Sci. USA. 103:2576-2581.

49. LIAVONCHANKA, A.; FEUSSNER, I. 2008. Biochemistry of PUFA Double Bond Isomerases Producing Conjugated Linoleic Acid. ChemBioChem. 9:1867-1872.

50. LOURENÇO, M.; RAMOS-MORALES, E.; WALLACE, R.J. 2010. The role of microbes in rumen lipolysis and biohydrogenation and their manipulation. Animal Feed Sci. Techn. 4(7):1008-1023.

51. MAIA, M.R.G.; CHAUDHARY, L.C.; FIGUERES, L.; WALLACE, R.J. 2007. Metabolism of polyunsaturated fatty acids and their toxicity to the microflora of the rumen. Antonie Van Leeuwenhoek. 91:303-314.

52. MAIA, M.R.G.; CHAUDHARY, L.C.; BESTWICK, C.S.; RICHARDSON, A.J.; McKAIN, N.; LARSON, T.R.; GRAHAM, I.A.; WALLACE, R.J. 2010. Toxicity of unsaturated fatty acids to the biohydrogenating ruminal bacterium, Butirivibrio fibrisolvens. BMC Microbiol. 10:52-62.
53. MARTIN, J.C.; VALEILLE, K. 2002. Conjugated linoleic acids: all the same or to everyone its own function? Reprod. Nutr. Dev. 42:525-536.

54. McKAIN, N.; SHINGFIELD, K.J.; WALLACE, R.J. 2010. Metabolism of conjugated linoleic acids and 18:1 fatty acids by ruminal bacteria: products and mechanisms. Microbiol. 156:579-588.

55. MOATE, P.J.; BOSTON, R.C.; JENKINS, T.C.; LEAN I.J. 2008. Kinetics of ruminal lipolysis of triacylglycerol and biohydrogenation of long-chain fatty acids: New insights from old data. J. Dairy. Sci. 91:731-742.

56. O'SHEA, M.; LAWLESS, F.; STATON, C.; DEVERY, R. 1998. Conjugated linoleic acid in bovine milk fat: a food-based approach to cáncer chemoprevention. Trends in Food Sci. \& Technol. 9:192-196.

57. PERFIELD, J.W.; LOCK, A.L.; GRIINARI, J.M.; SÆEBØ, A.; DELMONTE, P.; DWYER, D.A.; BAUMAN, D.E. 2007. Trans-9, cis-11 conjugated linoleic acid reduces milk fat synthesis in lactating dairy cows. J. Dairy Sci. 90: 2211-2218.

58. PRIETO, N.; DUGAN, M.E.R.; LÓPEZ-CAMPOS, Ó.; AALHUS, J.L.; UTTARO, B. 2013. At line prediction of PUFA and biohydrogenation intermediates in perirenal and subcutaneous fat from cattle fed sunflower or flaxseed by near infrared spectroscopy. Meat Sci. 94:27-33.

59. RIBEIRO, C.V.M.; EASTRIDGE, L.; FIRKINS, J.L.; STPIERRE, N.R.; PALMQUIST, D.L. 2007. Kinetics of fatty acid biohydrogenation in vitro. J. Dairy. Sci. 90:1405-1416.

60. ROSENFELD, I.S.; TOVE, S.B. 1971. Biohydrogenation of unsaturated fatty acids. IV. Source of hydrogen and stereospecificity of reduction. J. Biol. Chem. 246:5025-5030.

61. SACHAN, D.S.; DAVIS, C.L. 1969. Hydrogenation of linoleic acid by a rumen spirochete. J. Bacteriol. 98(1):300-301.

62. SHEN, X.; DANNENBERGER, D.; NUERNBERG, K.; NUERNBERG, G.; ZHAO, R. 2011. Trans-18:1 and CLA isomers in rumen and duodenal digesta of bulls fed n-3 and n-6PUFA-based diets. Lipids. 46:831841.

63. SHINGFIELD, K.J.; LEE, M.R.F.; HUMPHRIES, D.J.; 
SCOLLAN, N.D.; TOIVONEN, V.; REYNOLDS, C.K.; BEEVER, D.E. 2010. Effect of incremental amounts of fish oil in the diet on ruminal lipid metabolism in growing steers. Brit. J. Nutr. 104:56-66.

64. SINGH, S.; HAWKE, J.C. 1979. The in vitro lipolysis and biohydrogenation of monogalactosyldiglyceride by whole rumen contents and its fractions. J. Sci. Food Agric. 30:603-612.

65. STERK, A.; HOVENIER, R.; VLAEMINCK, B.; VAN VUUREN, A.M.; HENDRIKS, W.H.; DIJKSTRA, J. 2010. Effects of chemically or technologically treated linseed products and docosahexaenoic acid addition to linseed oil on biohydrogenation of C18:3n-3 in vitro. J. Dairy Sci. 93:5286-5299.

66. TROEGELER-MEYNADIER, A.; NICOT, M.C.; BAYOURTHE, C. 2003. Effects of $\mathrm{pH}$ and concentration of linoleic and linolenic acid on extent and intermediates of ruminal biohydrogenation in vitro. J. Dairy. Sci. 86:4054-4063.

67. VÁRADYOVÁ, Z.; KIŠIDAYOVÁ, P.S.; DUŠAN, J. 2008. Comparison of fatty acid composition of bacterial and protozoal fractions in rumen fluid of sheep fed diet supplemented with sunflower, rapeseed and linseed oils. Anim. Feed. Sci. Technol. 144:44-54.

68. WALLACE, R.J.; MCKAIN, N.; SHINGfiELD, K.J.; DEVILLARD, E. 2007. Isomers of conjugated linoleic acids are synthesized via different mechanisms in ruminal digesta and bacteria. J. Lipid. Res. 48:22472254.

69. WRIGHT, D.E. 1959. Hydrogenation of lipids by rumen protozoa. Nature. 184:875-876.

70. WRIGHT, D.E. 1960. Pectic enzymes in rumen protozoa. Arch. Biochem. Biophys. 86:251-254.

71. YAMAZAKI, S.; TOVE, S.B. 1979. Biohydrogenation of unsaturated fatty acids. Presence of dithionite and an endogenous electron donor in butyrivibrio fibrisolvens. J. Biol. Chem. 254(10):3812-3817.

72. YAÑEZ-RUIZ, D.R.; SCOLLAN, N.D.; MERRY, R.J.; NEWBOLD, C.J. 2006. Contribution of rumen protozoa to duodenal flow of nitrogen, conjugated linoleic acid and vaccenic acid in steers fed silages differing in their water-soluble carbohydrate content. Br. J. Nutr. 96:861-869.

73. ZENED, A.; ENJALBERT, F.; NICOT, M.C.; TROEGELER-MEYNADIER, A. 2013. Starch plus sunflower oil addition to the diet of dry dairy cows results in a trans-11 to trans-10 shift of biohydrogenation. J. Dairy Sci. 96:451-459.

Recibido: Septiembre 26 de 2012

Aceptado: Junio 4 de 2013

Como citar:

Castillo V., J.; Olivera A., M.; Carulla F., J. 2013. Descripción del mecanismo bioquímico de la biohidrogenación en el rumen de ácidos grasos poliinsaturados: una revisión. Rev. U.D.C.A Act. \& Div. Cient. 16(2): 459-468. 